\title{
Emerging Paradigm-Shifting in Cancer Immunotherapeutic Towards Personalized Cancer Medicine and Potential Challenges
}

\author{
Bhuvnesh K Sharma, PhD* \\ Senior Director, R\&D (Translational Oncology), ScyTek Labs, Logan UT USA
}

ISSN: 2637-773X

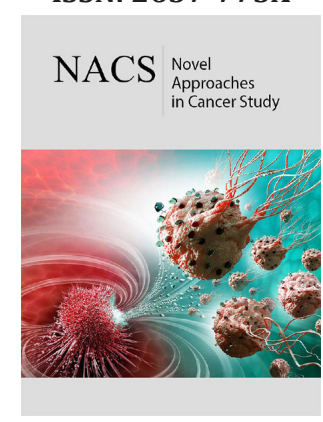

*Corresponding author: Bhuvnesh K Sharma PhD, Senior Director, R\&D (Translational Oncology), ScyTek Labs, 205 South $600 \mathrm{~W}$, Logan UT, USA, email: bkumar111@hotmail.com,

Phone: +14359942823

Submission: 嗑: September 21, 2020

Published: 望 November 13, 2020

Volume 5 - Issue 4

How to cite this article: Bhuvnesh $\mathrm{K}$ Sharma. Emerging Paradigm-Shifting in Cancer Immunotherapeutic Towards Personalized Cancer Medicine and Potential Challenges. Nov Appro in Can Study. 5(4). NACS.000616. 2020. DOI: 10.31031/NACS.2020.05.000616

Copyright@ Bhuvnesh K Sharma, PhD, This article is distributed under the terms of the Creative Commons Attribution 4.0 International License, which permits unrestricted use and redistribution provided that the original author and source are credited.

\begin{abstract}
Recently invented several cancer immunotherapies that have succeeded with a diversity of new agents for clinical use. As such, notable progress immensely facilitated the designing of numerous effective immunotherapeutic regimens. Therapeutic vaccines and immune checkpoint blockade have lately demonstrated an enormous therapeutic efficacy thus generated great excitement for the development of new avenues on cancer treatments. Immunotherapies are highly potential to generate tumor-specific responses, as well as maintaining long-lasting remissions, unraveling vital approaches for future discoveries of cancer medicine. Next-generation cancer immunotherapies engaged with native mono-bispecific antibodies are intended to target at innate immune checkpoints, conditional designing activated immune stimulator, redirecting Innate Cell Engagers (ICEs). Similarly, Natural killer cells are engineered for multi-specific immune targeting utilizing adaptable affinities and avidities, redirecting innate immune cells (dendritic, macrophages \& NK cells). Oncolytic adenovirus-mediated targeting to alter cold tumors into hot including challenging tumor heterogeneity via interfering tumor sub-clonality are attractive projections to develop several novels immune-oncologic therapeutics towards personalized cancer medicine.
\end{abstract}

\section{Introduction}

Cancer is a leading cause of death worldwide. Despite enormous unraveling, the mysterious nature of this disease, as well as massive advancement in the management technologies, the exploration of effective cancer treatment remains a far-distant dream. Due to this fact, there is a steady global growth of cancer morbidly and mortality possibly linked with the aging population including increasingly embracing of detrimental lifestyles. This escalating morbidity incidence reflects that the existing empirical therapeutic options viz. chemotherapy, radiation therapy, and surgical intervention are consistently failed to control tumor growth or relapse. Such perturbing trends suggest an urgency to revisit our strategies on our achievable modalities of cancer treatment and to implement next-generation technologies in future diagnostic, prognostic, and therapeutic strategies for cancer. Current cutting-edge research immensely revolutionized efficacious treatment options-chemotherapeutics, molecularly targeted agents thus there is great optimism in the development of immunotherapeutic which holds significant potential in treatment regimens.

In recent decades, we have encountered numerous shortcomings of traditional cancer therapies as $90 \%$ of new cases drugs failed in the clinic. Recent innovative therapeutics have reflected a swing from conservative cancer management towards personalized medicine. However, there is a growing optimism for innovative treatment strategies aiming to cure cancer and to prolong survival as well as the highest probable quality of life during treatment. The future of cancer treatment lies in providing patients with an even greater level of personalization.

\section{Personalized cancer medicine}

Personalized Cancer Medicine is now at the most crucial phase in its conventional management to personalized cancer treatment strategies. However, there are numerous hurdles in the advancement of personalized cancer medicine. Several clinical data delineated signatures associated with response to immunotherapy and key mechanisms modulating immunotherapeutic response. The Cancer Immunotherapy arena is swiftly making great strides with therapeutic options in personalized cancer medicine. 


\section{New advances and treatment strategies}

New advances and treatment strategies are unraveling the power of the immune system in accomplishing the robust potential of immune oncology. Monoclonal antibody drugs are a fair innovation in the treatment of various human cancers. Some monoclonal antibody drugs could be used in combination with other therapeutic options such as chemotherapy and hormone therapy. Recently several genetically engineered monoclonal antibodies have been developed to attach and targeting certain antigenic epitopes of cancer cells. The monoclonal antibody drugrituximab (Rituxan) attaches to a specific protein (CD20) exclusively expressed on B-Cell, a subset of white cells specifically utilized to treat a certain type of lymphomas arising from B-cells [1,2].

\section{Interventional checkpoint inhibitors}

Interventional checkpoint inhibitors are emerging forefront cornerstone of current cancer immunotherapies encompassing a wide range of cancers that respond to treatment. With a deeper understanding of the immunobiology as well as their signatures, neo-epitopes are linked with checkpoint therapy. These checkpoint inhibitors are the first of a new generation of immunotherapy treatments, revolutionizing treatment, increasing efficacy and response rates, for many different types of cancers. It is now becoming possible to prime the immune system utilizing combining checkpoint blockade therapies with agents that are toxic to the cancer cells. However, the trick lies in carefully choosing the right drugs, given at the right time, for their true optimization and effectiveness. Various combinations of checkpoint inhibitors are being designed and /or tested such as double checkpoint blockade, combination with chemotherapy, radiotherapy, molecularly targeted agents, and other immune directed strategies [3].

\section{Combinational cancer immunotherapy}

There is a mounting consensus that combinational therapies including vaccination may facilitate clinical benefit. Conscious efforts are being made to utilize combinational immunotherapies for the advance stage of the disease Various combinations of checkpoint inhibitors are being designed and /or tested such as double checkpoint blockade, combination with chemotherapy, radiotherapy, molecularly targeted agents, and other immunedirected strategies [4].

\section{Several investigational combinational immunotherapies}

Several investigational combinational immunotherapies that target novel immune checkpoint PD-1/PDL1 and Cytotoxic T-lymphocytes (CTLA-4), CD27, CD80, CD86, CD20, and IDO therapies are being widely explored in many pre-clinical and clinical trials of solid tumors. This could enhance drug efficacy in achieving durable remission in the disease. Tumor-associated macrophages express PD-1 thus suggest that PD1/PDL1 therapies may have a direct effect on macrophages $[5,6]$.

\section{Two anti-PD-1 antibodies}

Two anti-PD-1 antibodies such as -Keytruda for Metastatic Non-small Cell Lung Cancer and Nivolumab (Opdivo) for several cancers that are metastatic (such as lung cancer, melanoma, and colorectal cancer) are now approved by US-FDA. However, two clinical trials with both nivolumab (Opdivo) and pembrolizumab (Keytruda),facilitated the conception that those patients whose tumors have higher levels of PD-1 on their surface are likely a better responder to $\mathrm{PD}-1$ treatment in comparison with patients whose tumors have low or no PD-L1 expression.Further,PD-1 blockade treatment strategies in combination with another agent seems to be more promising than a single regime. There is a notion that those patients whose tumors have higher levels of PD-L1 on their surface are more likely to respond to anti-PD-1 treatment compared to those patients whose tumors have low or no PD-L1 expression. AstraZeneca, which is now developing durvalumab / MEDI4736, an anti-PD-L1 immunotherapy antibody. It is anticipating that checkpoint blockade combination comes to the lead, extending the range of cancers that respond to treatment, besides increasing efficacy and response rates.

Another progressive candidate in the pipeline targets CD47 which acts as inhibitors of phagocytosis of healthy cells. CD47 is often overexpressed on cancer cells which protects tumors from phagocytosis thus providing an opportunity to develop drugs that restore the phagocytic activity of macrophages. Therefore, a combinational blockade of PD-1 and CD47 has been shown to enhance antitumor effects in-vivo which presents an interesting opportunity for combinational therapeutic strategic options. Therefore, the combinational cancer therapeutics approach may facilitate treatment responsiveness among cancer patients [6].

\section{Chimeric antigen receptor-T cells (CAR-T cells)}

Chimeric antigen receptor-T cells (CAR-T cells) are novel tools of innovative therapy transitioning to focus on solutions that are being developed for solid tumors. It is a more promising area of cancer treatment, in particular, targeting various blood and solid cancers. CAR-T cell therapy has shown impressive clinical results. CAR T-cell therapies to edit out genes that inhibit $\mathrm{T}$ cells, such as PD-1, or to introduce genes that code for the expression of certain cytokines or chemokine receptors. Engineering nonimmune cells, including cells outfitted with "synthetic T-cell receptor-like signal transduction devices, can increase efficacy in off-the-shelf CAR-T therapies [7]. There are some reports that CAR-T cells can secret powerful immunostimulatory cytokines upon activation of specific small molecules. CAR -T cell cancer immunotherapies has also shown an impressive clinical result, in particular, targeting various blood and solid cancers. The main drawback of this therapy is the non-existence of the preclinical model to appropriately evaluate the potential lethal toxicity $[8,9]$.

\section{Adoptive cell cancer immunotherapy}

Adoptive Cell Cancer Immunotherapy is another spectacular strategy that involves reinfusing a patient's immune cells after they have been engineered in the laboratory to recognize cancer-specific killer T-cells and destroy the patient's specific cancer in some blood cancers associated with the lymphoid system. Although, this immunotherapeutic seems to be less efficacious in solid 
tumors but it opens up a new opportunity for improving cancer immunotherapy [10].

\section{Antibody-drug conjugates (ADC) cancer immunotherapy}

Some European Biotech/ Pharma companies now striving to develop several antibody-drug conjugates by exploiting a vast repository of numerous novel compounds as cytotoxic payload with monoclonal antibodies to automate cancer drug designs [11].

Dendritic Cell (DCs) based Cancer Immunotherapy are pivotal players in initiating immune responses thus contemplating their exploration in cancer immunotherapy. A powerful approach to cancer therapy involves modifying the patient's immune cells to enhance the immune response against cancer cells. The most studied cell types for cancer immunotherapy are dendritic cells (DCs) and T cells, which are crucial players in initiating immune responses. DCs are increasingly used in vaccine research as immunotherapy for cancer and other diseases. DCs are the most potent and proficient antigen-presenting cells and are critical to the onset of immunity. They have the highest surface density of major histocompatibility complex (MHC) and costimulatory molecules, together with high motility, which allows them to transport from the site of antigen uptake to the lymph node, thus facilitating to produce immunostimulatory cytokines and chemokines [12].

\section{Macrophage directed therapies}

Macrophage directed Therapies are recent inventions to rival PD1/PDL1 checkpoint inhibitors and T- cell approaches in solid tumors. Various approaches from CD47 blockades to CAR macrophages may provide opportunities for newer cancer drug development. However, there are several challenges to control the fluctuating phenotype of macrophage cells and improve their efficacy with combination cancer therapies [13].

\section{Bi- or Multi specific antibody therapy}

There is another growing optimistic immunotherapeutic approach on bi- and multi-specific antibody (bsAb) technology as a possible next generation of targeted biologics for cancer therapy. Recent cutting-edge escalating technologies on the utilization of the sequence and configuration of antibodies have greatly facilitated researchers to expand the therapeutic potential of monoclonal antibodies immensely. Search for Bispecific antibodies in cancer therapies are being employed as costimulatory agents of $\mathrm{T}$ cells to facilitate regulating the immune response to various tumors. Tremendous progress in bsAb research and the regulatory success of two bispecific molecules has led to an "explosion" of T-cell redirecting bsAbs [14]. Numerous recently developed mono-or bi-specific antibodies are now plausible attractive tools for nextgeneration combinational therapeutic modalities.

\section{Targeted oncolytic immunotherapies}

Targeted Oncolytic Immunotherapies are hottest exploration of the recent decades on targeted Immunotherapies. This is a keystone therapeutic modality for oncology which is likely to generate potential avenues implementing into the clinic. It would also facilitate developing strategies into a systemic un-invasive delivery system that can travel intravenously to the tumor site and overcome the immune system response [15].

\section{T-Regulatory (T-regs) cells in Cancer treatment}

Recently, the role of T-reg in cancer treatment, suggests that the precise action of T-regs using molecular approaches could help enhance the efficacy of cancer immunotherapy drugs unleashing the immune system against tumors [16].

\section{Epigenetic cancer drug discovery}

Reversibility of epigenetics modifications accelerated efforts to develop epigenetic therapeutics. Targetable epigenetics modifications and the number of medical conditions with which they are linked appears to be on the horizon [17].

\section{Potential challenges in applications of cancer immunotherapeutic}

Despite numerous existing cellular onco-immunotherapies, with their considerable efficacy, their applications are limited with significantly higher incidences of toxicities and limited implementation. Recently developed immunotherapies are highly efficacious in some patients. However, many patients are unresponsive or sometimes suffer from acute inflammation-related adverse immune reactions.

Additionally, a high failure rate of immunotherapies is also encountered in their clinical validations. Regardless of innumerable challenges, pharmaceutical industries are also advancing their strategies to design and develop the most effective cancer immunotherapeutic. Many patients are unresponsive or suffer from inflammation-related adverse immune reactions. Such streamlined development requires rigorous pre-clinical and clinical validation for treating cancer patients. The future development of effective and safe immunotherapies will depend on our capability to anticipate and diminish immune-related adverse events.

Upon administering CAR therapy, CAR T cells proliferate, there's a higher chance of having neurologic toxicity or Cytokine release syndrome (CRS), Diarrhea, colitis, increased lipase, and increased ALT and AST levels. There is an understood risk that CAR-T cells may cross-react with normal antigens on noncancerous cells and damage healthy tissue as - Off-target effect. Hence general risk assessment for CAR T-cell therapies should be taken into consideration before administering this therapeutic strategy.

\section{Adverse effects of cancer immunotherapies}

The effectiveness of immunotherapy-related side effects is less predictable in comparison with other types of cancer management strategies Most of the known immunotherapies are envisioned to minimize side effects and improve the quality of life. However, a majority of the adverse effects are mild to moderate in severity. Administration of immunotherapy may develop side effects immediately after or long after a course of treatment receiving the first dose. Patients receiving certain immunotherapy drugs- such as checkpoint inhibitors drug, pembrolizumab (Keytruda)should "be closely monitored" to save from life-threatening complications or 
early diagnosis and treatment. These therapies may cause immune cells to attack healthy cells, which cause a variety of common side effects, including skin reactions at the site of the injection such as fatigue, nausea, diarrhea, and flu-like symptoms. pain, swelling, and soreness. Some immunotherapy drugs may cause severe or even fatal allergic reactions, though this is rare. Such complications frequently leading to the discontinuation of therapy or may cause severe health damage to the patient. Such side effects are used by immune checkpoint inhibitions (ICI) immune-related adverse events ranging from non-threatening conditions such as skin rash to more serious complications involving the joints, endocrine glands, lungs, liver, and other vital organs. Although some of the side effects could be fixed with additional medication there is a strong possibility of irreversible effects that would require longterm health care.

Several rare side effects such as colitis, hepatitis, inflammation of the lung, or pneumonitis, kidney failure, myocarditis or inflammation of the heart, neuropathy, paralysis, meningitis, or encephalitis, pancreatitis, severe infections, may reflect a life-threatening condition which may require an immediate medical intervention. Fortunately, in a majority of cases, probable immunotherapy-related side effects can be managed safely with immunosuppressive drugs such as steroids as long as the potential side effects are identified and treated early.

\section{Pitfalls / missing links}

Immunotherapy is impacting each cancer in different ways. The effectiveness of Immunotherapies depends on molecular architecture and presentation of components. So far, no unified/ comprehensive or individualized treatment protocol utilizes the most modern, and cutting-edge medications, treatments and administering immunotherapies for various types of cancers. Although several ongoing clinical trials have clearly shown a better efficacy of immunotherapy and fewer side effects in patients treated with various types of cancer Immunotherapeutic, many questions are still unanswered. For example, how to better modify genetic mutation-based the treatment.

Recently identified several clues have suggested that cold tumors are deficient in primary immunological response in particular T cell priming and expansion. This problem is not only linked with exhausted $\mathrm{T}$ cells or $\mathrm{T}$ cells that do not penetrate tumor stroma but possibly $\mathrm{T}$ cells have not been adequately generated in the first place. Current efforts are being focused on "agonist therapy" to generate and amplify T cell responses. These efforts include CAR $\mathrm{T}$ cells, second-generation cancer vaccines. In addition, unique treatment strategies are advancing further based on next-generation genetic testing which is the key to the successful utilization of a targeted method to deliver microdoses of genetically determined immunotherapies.

Ithas been postulated that there is a high level of nonsynonymous mutational load and molecular subtypes lead to conformational changes affecting their poor response to immunotherapies as well as also not generating adequately effector $\mathrm{T}$ cells thus losing their capability to penetrate tumor stroma. Further, checkpoint inhibitors are effective only in a subset of patients. Tumor cells themselves can orchestrate $\mathrm{T}$ cell exclusion, for example by oncogene-driven alterations in chemokine production that recruit suppressive myeloid cells. Oncogene-driven upregulation of tumor PD-L1 contributes to immunosuppression. Exploration of altering cold tumors into hot tumors with a high degree of $\mathrm{T}$ cell exclusion, blocking only PD-1 or CTLA-4 may be insufficient to achieve a clinical response if other suppressive pathways such as IDO, CD73, TIGIT, VISTA, are also expressed and function in a non-redundant manner irrespective blockade of PD-1 or CTLA-4 pathway.

Certain critical factors such as toxicity, dosing, and sequencing issues which are inked with the evolving combinational immunotherapy, still remains challenging. In addition, other vital factors such as agonists, immune pharmacodynamic need to be synergized with currently approved immune therapies in particular checkpoint blockade antibodies. Further, engineering CAR to comprise innovative building blocks or intrinsic alterations may possibly resolve the issues of hypoxia, T-Regulatory cells, and intratumorally migration. Since Checkpoint inhibitors are effective only in a subset of patients hence it is necessary to redesign achieving personalized approaches.

\section{Future paradigm of cancer immunotherapies}

Personalized cancer medicine is at the most vital phase in its development. We are now anticipating explosive growth for immunotherapies over the next years. However, the future of immunotherapies will depend on the quality of clinical trial data. Besides, the industry is continuing to work on a standardized approach to proficiently and reproducibly level of patient immune profile and developing biomarkers that predict response to treatment. The discovery of newer surrogate cancer markers could provide an avenue to new immunotherapies and personalized cancer care. Newer innovative initiatives on in-vivo autoimmunization by intralesional of low nontoxic doses of GM-CSF and IL-2 prior to its surgical resection can utilize each patient tumor as a source for tumor-specific antigenic or genetic profiles [18-21]. This approach activates autologous DCs and Cytotoxic T-cells (CTL) in-vivo and can overcome tumor heterogeneity and also confers autogenic and tumor-specific therapy, However, controlled clinical trials would require to authenticate this hypothesis on their beneficial effects.

Recent strategies on the rational selection of cancer immunotherapeutic are becoming more focused on improving patient's outcome by offering custom-made targeted treatments to an individual's predisposing molecular profile of pathological lesions. This is the most important requirement of a clinical evaluation of effective cancer immunotherapy.it is now becoming possible to prime the immune system, but the trick is in carefully choosing the right drugs, given at the right time, to truly optimize effectiveness.

\section{Concluding Remarks}

Rapidly evolving genomic technology and our understanding of the molecular basis of disease and health began to deliver on 
their promise of more effective targeted therapeutics. Despite innumerable challenges, pharmaceutical industries are enormously advancing in making the most effective and efficient cancer immunotherapeutic. in-depth genomic analysis in context with tumor and immune cells will help to prioritize the development of new treatments and immunotherapies for a wide range of cancer.

Next-generation cancer immunotherapies with native monobi-specific antibodies targeting innate immune checkpoints, conditional designing activated immune stimulator, redirecting innate cell engagers (ICEs), natural killer cells for immune targeting to multi-specific tumor targets utilizing modifiable affinities and avidities, redirecting innate immune cells Oncolytic adenovirusmediated turning cold tumors hot including challenging tumor heterogeneity via interfering tumor sub-clonality are attractive predictions to develop immune-oncologic therapeutics thus facilitating to advance novel therapies towards personalized cancer medicine.

However, before exploring the development of newer cancer immunotherapy, it would be essential to take into the consideration a comprehensive ramification on immune cell functioning beyond the lymphoid system so that a precise personalized cancer-fighting immunotherapies strategy could be explored.

\section{References}

1. John M, Lambert JM (2005) Drug-conjugated monoclonal antibodies for the treatment of cancer. Current Opinion in Pharmacology 5(5): 543549 .

2. Trail PA, Bianchi AB (1999) Monoclonal antibody-drug conjugates in the treatment of cancer. (A Review) Current Opinion in Immunology 11(5): 584-588.

3. Postow MA, Callahan MK, Wolchok JD (1982) Immune checkpoint blockade in cancer therapy. J Clin Oncol 33(17): 1974-1982.

4. Danielle Lussier, John L Johnson, Pooja H, Joseph Blattman (2015) Combination immunotherapy with $\alpha$-CTLA-4 and $\alpha$-PD-L1 antibody blockade prevents immune escape and leads to complete control of metastatic osteosarcoma. Journal for Immunotherapy of Cancer 3: 21.

5. Karamouzis MV, Papavassiliou (2018) Combination of checkpoint inhibitors with other agents as a strategy to improve anti-cancer effect-a glimpse to the future. Expert Opinion Invest Drugs 27(7): 569-572.

6. Anagnostou VK, Brahmer JR (2015) Cancer immunotherapy: A future paradigm shift in the treatment of non-small cell lung cancer. Clin Cancer Res 21(5): 976-984.
7. Pagel JM, West H (2017) Chimeric Antigen Receptor (CAR) T cell therapy. JAMA Oncol 3(11): 1595.

8. Morgan RA, Yang JC, Kitano M, Dudley ME, Laurencot CM, et al. (2010) Case report of a serious adverse event following the administration of $\mathrm{T}$ cells transduced with a chimeric antigen receptor recognizing $\mathrm{ERBB}_{2}$. Mol Ther 18(4): 843-851.

9. Bonifant CL, Jackson HJ, Brentjens RJ, Curran KJ (2016) Toxicity, and management in CAR T-cell therapy. Molecular Therapy - Oncolytics 3: 16011.

10. Jiang X, Jiang Xu, Mingfeng LM, Xing H, Wang Z, et al. (2019) Adoptive CD8+ $\mathrm{T}$ cell therapy against cancer: Challenges and opportunities. Cancer Letters 462: 23-32.

11. Nejadmoghaddam M, Arash Minai-Tehrani, Ramin Ghahremanzadeh, Morteza Mahmoudi, Rassoul Dinarvand, et al. (2019) Antibody-drug conjugates: Possibilities and challenges. Avicenna J Med Biotech 11(1): 3-23.

12. Bol KF, Schreibelt G, Rebold K, Wculek SK, Julia K, et al. (2019) The Clinical application of cancer immunotherapy based on naturally circulating dendritic cell. J Immunother Cancer 7(1): 109.

13. Schubert GS (2014) Macrophage activation syndrome, and cytokine directed therapies. Best Pract Res Clin Rheumatol 28(2): 277-292.

14. Runcie K, Budman DR, John V, Seetharamu N (2018) Bi-specific and trispecific antibodies- the next big thing in solid tumor therapeutics. Mol Med 24(1): 50.

15. Raja Ji, Ludwig JM, Gettinger SN, Schalper KA, Hyun Kim (2018) Oncolytic virus immunotherapy: prospects for oncology. Immunotherapy Cancer 6(1): 140 .

16. Shitara K, Nishikawa H (2018) Regulatory T cells: A potential target in cancer immunotherapy. Ann N Y Acad Sci 1417(1): 104-115.

17. Ahuja N, Anup Sharma, Baylin SB (2016) Epigenetic therapeutics: A new weapon in the war against cancer. Annual Rev Med 67: 73-89.

18. Emens LA, Butterfield LH, Hodi S, Marincola FM, Kaufman HL (2016) Cancer Immunotherapy trials: Leading a paradigm shift in drug development. J Immunotherapy Cancer 4: 42.

19. Elias EG (2012) A new approach to adjuvant therapy in high-risk melanoma patients. ASCO Annual Meeting. E1;3129.

20. Elias EG, Sharma, BK (2013) Targeting melanoma sites in-vivo; can induce complete tumor ablation and prolong patients' survival: An exploratory study. J Cancer Sci Ther 5: 244-248.

21. Elias G, Sharma, BK (2014) Adjuvant therapy in high-risk patients with primary cutaneous melanoma: Past, present, and future. Aperito J Cancer Studies 12(7): 106. 